\title{
Partitioning the impact of environment and spatial structure on alpha and beta components of taxonomic, functional, and phylogenetic diversity in European ants
}

Xavier Arnan, Xim Cerdá, Javier Retana

We analyze the relative contribution of environmental and spatial variables to the alpha and beta components of taxonomic (TD), phylogenetic (PD), and functional (FD) diversity in ant communities found along different climate and anthropogenic disturbance gradients across western and central Europe, in order to assess the mechanisms structuring ant biodiversity. To this aim we calculated alpha and beta TD, PD, and FD for 349 ant communities, which included a total of 155 ant species; we examined 10 functional traits and phylogenetic relatedness. Variation partitioning was used to examine how much variation in ant diversity was explained by environmental and spatial variables. Autocorrelation in diversity measures and each trait's phylogenetic signal were also analyzed. We found strong autocorrelation in diversity measures. Both environmental and spatial variables significantly contributed to variation in TD, PD, and FD at both alpha and beta scales; spatial structure had the larger influence. The different facets of diversity showed similar patterns along environmental gradients. Environment explained a much larger percentage of variation in FD than in TD or PD. All traits demonstrated strong phylogenetic signals. Our results indicate that environmental filtering and dispersal limitations structure all types of diversity in ant communities. Strong dispersal limitations appear to have led to clustering of TD, PD, and FD in western and central Europe, probably because different historical and evolutionary processes generated different pools of species. Remarkably, these three facets of diversity showed parallel patterns along environmental gradients. Trait-mediated species sorting and niche conservatism appear to structure ant diversity, as evidenced by the fact that more variation was explained for FD and that all traits had strong phylogenetic signals. Since environmental variables explained much more variation in FD than in PD, functional diversity should be a better indicator of community assembly processes than phylogenetic diversity. 
6 1Departamento de Botânica, Universidade Federal Pernambuco, Av. Prof. Moraes Rego s/no,

7 Cidade Universitária, 50670-901, Recife, PE, Brazil

8 2Estación Biológica de Doñana, CSIC, Avda. Américo Vespucio, s/n, E-41092 Sevilla, Spain

9 3Univ. Autònoma Barcelona, Cerdanyola del Vallès, 08193 Catalunya, Spain

$10 \quad{ }^{4}$ CREAF, Cerdanyola del Vallès, 08193 Catalunya, Spain

11

12

*Corresponding author. Email: x.arnan@creaf.uab.es 


\section{ABSTRACT}

We analyze the relative contribution of environmental and spatial variables to the alpha and beta components of taxonomic (TD), phylogenetic (PD), and functional (FD) diversity in ant communities found along different climate and anthropogenic disturbance gradients across western and central Europe, in order to assess the mechanisms structuring ant biodiversity. To this aim we calculated alpha and beta TD, PD, and FD for 349 ant communities, which included a total of 155 ant species; we examined 10 functional traits and phylogenetic relatedness. Variation partitioning was used to examine how much variation in ant diversity was explained by environmental and spatial variables. Autocorrelation in diversity measures and each trait's phylogenetic signal were also analyzed. We found strong autocorrelation in diversity measures. Both environmental and spatial variables significantly contributed to variation in TD, PD, and FD at both alpha and beta scales; spatial structure had the larger influence. The different facets of diversity showed similar patterns along environmental gradients. Environment explained a much larger percentage of variation in FD than in TD or PD. All traits demonstrated strong phylogenetic signals. Our results indicate that environmental filtering and dispersal limitations structure all types of diversity in ant communities. Strong dispersal limitations appear to have led to clustering of TD, PD, and FD in western and central Europe, probably because different historical and evolutionary processes generated different pools of species. Remarkably, these three facets of diversity showed parallel patterns along environmental gradients. Trait-mediated species sorting and niche conservatism appear to structure ant diversity, as evidenced by the fact that more variation was explained for FD and that all traits had strong phylogenetic signals. Since environmental variables explained much more variation in FD than in PD, functional 
37 diversity should be a better indicator of community assembly processes than phylogenetic 38 diversity. 
INTRODUCTION

41 A central goal in ecology is to describe patterns of species diversity and composition along broad

42

43 environmental gradients and to identify the mechanisms that underlie them (e.g., Pianka, 1966; Gaston, 1996). For instance, broad-scale patterns of species richness are often correlated with contemporary climate (Gaston, 1996). However, since climatic factors are usually correlated with latitude, and latitude is, in turn, correlated with other factors, the underlying causes of variation in species richness are much debated (e.g., Pianka, 1966; Willig et al., 2003). Moreover, other environmental factors besides (or in addition to) climate can affect diversity patterns. For instance, species richness and composition may vary along disturbance (Fox, 2013) or habitat heterogeneity gradients (Rahbek et al., 2007).

Spatial factors might also influence diversity patterns. In fact, environmental gradients are themselves spatially structured (Legendre \& Legendre, 1998), and random but spatially limited dispersal of species (Tuomisto et al., 2003) can also generate spatially structured patterns. Consequently, dispersal limitations and habitat and environmental similarities may result in positive spatial autocorrelation in communities (Legendre et al., 2009). In particular, the extent to which species diversity patterns are determined by environmental filters versus random but spatially autocorrelated dispersal are intensely debated (e.g., Tuomisto et al., 2003). It has been suggested that simultaneously examining the influence of environmental and spatial factors on communities could reveal their relative importance (e.g., Borcard et al., 2004). If species diversity patterns solely vary along environmental gradients, it would indicate that the underlying mechanism is environmental filtering; if only spatial structure has an effect, variation in diversity patterns may arise from dispersal limitations. Although there has been a recent increase in the number of studies analyzing the relative contribution of environmental and spatial 
63 factors to species diversity patterns, taxonomic diversity (TD) has been the main focus; other

64 diversity components have only rarely been examined (but see Meynard et al., 2011).

65 The problem is that measures of TD treat all species as evolutionarily independent and

66 ecologically equivalent and therefore may not provide enough information regarding the

67 mechanisms underlying community patterns. For this reason, new biodiversity metrics that

68 incorporate information about the functional and phylogenetic characteristics of communities

69 have recently been proposed. While phylogenetic diversity (PD) reflects the accumulated

70 evolutionary history of a community (Webb et al., 2002), functional diversity (FD) reflects the

71 diversity of morphological, physiological, and ecological traits found therein (Petchey \& Gaston,

72 2006). Understanding how PD and FD relate to TD can provide insights into the extent to which

73 community assembly is driven by deterministic versus stochastic processes (Cavender-Bares et al., 2009; Pavoine \& Bonsall, 2011; Purschke et al., 2013). For a given phylogeny of available

75 lineages and evolutionary rate for functional traits, one would expect to see different patterns of phylogenetic and functional community structure depending on whether competition or environmental filtering is the primary driver of community assembly (Webb et al., 2002; Kraft et al., 2007). It is generally thought that FD and PD are positively correlated with TD at the regional scale (e.g., Forest et al., 2007; Faith, 2008; Meynard et al., 2011; but see Losos, 2008; Devictor et al., 2010; Safi et al., 2011). If functional traits allow species to locally adapt to environmental conditions (Pavoine et al., 2011), it may be that environmental filters predominantly influence the functional structure of communities and that nothing is reflected by their taxonomic and phylogenetic structures (Díaz et al., 2007; Mouchet et al., 2010). However, 84 a strong correlation between FD and PD would be expected if the functional traits that allow 
species to persist in the environment are evolutionarily conserved, that is to say, they display phylogenetic signals (Webb et al., 2002; Cavender-Bares et al., 2009).

Since the processes that shape biodiversity differ across scales (Whittaker et al., 2001), it is also relevant to study the aforementioned facets of diversity at different scales of analysis (e.g., alpha and beta diversity) (Devictor et al., 2010; Bernard-Verdier et al., 2013). Analyzing patterns at only one of these scales can result in misleading or incomplete interpretations of the results (Whittaker et al., 2001). For instance, if environmental determinism is at work via trait-based species sorting, significant patterns of turnover (i.e., beta diversity) in FD, PD (assuming niche conservatism), and TD will be found. In contrast, it may not translate into significant patterns in alpha-level TD, PD, and FD: their values may remain unchanged despite significant species, species-trait, or lineage turnover (Mouchet et al., 2010). In addition, addressing both scales of analysis provides complementary information. At the local-level, biotic interactions, environmental filtering, and stochastic processes play major roles in determining (alpha) diversity whereas, at more regional scales, environmental filtering as well as historical and evolutionary processes may largely drive (beta) diversity (Cavender-Bares et al., 2009). For instance, it is thought that environmental filtering operates more strongly at the regional scale (Cornwell et al., 2006), while species interactions (e.g., competition) drive local-level assembly patterns (Cavender-Bares et al., 2004; Slingsby \& Verboom, 2006).

In this study, we evaluated how alpha and beta TD, PD, and FD in ant communities across western and central Europe are shaped by environmental and spatial factors, with the aim of understanding the mechanisms that structure communities. We also analyzed trait phylogenetic signals to determine if they helped shape pattern similarity among the three facets of diversity. Ants are a good study system when it comes to examining biodiversity patterns because they are 
108

109

110

111

112

113

114

115

116

117

118

119

120

121

122

123

124

125

126

127

128

129

among the most diverse and abundant organisms on earth and perform a great variety of ecological functions that are critical for ecosystems (Hölldobler \& Wilson, 1990). We compiled data from 349 ant communities that included a total of 155 ant species, and we characterized a set of 10 functional traits that reveal different dimensions of the ant functional niche. We also quantified ant phylogenetic relatedness. Our communities were distributed along different broad environmental gradients (e.g., climate, land-use, and anthropogenic disturbance). This is the first study to analyze the relative contribution of different factors to different facets of biodiversity at two different scales (alpha and beta). We generated the following three hypotheses. First, environmental heterogeneity and space should significantly contribute to variation in TD, PD, and FD at both alpha and beta scales. However, since ants face dispersal limitations (Mezger \& Pfeiffer, 2011), we expected spatial factors to make a larger contribution. Second, the different facets of biodiversity should show similar responses along environmental gradients. Third, because environmental filtering is expected to limit community members to those that are preadapted to local conditions, and that are thus functionally similar, environmental factors should influence FD more than TD or PD; in turn, if strong niche conservatism exists, PD and FD should display similar patterns.

\section{MATERIAL AND METHODS}

\section{Ant community data}

Information on the species composition of European ant communities was obtained from primary data collected by the authors and from an exhaustive search of scientific literature that contained species abundance or presence-absence data from single locations. The dataset encompassed 349 
130 sites from eleven different countries (Figure 1). At these sites, there were a total of 155 ant

131 species (Table S1). As abundance information does not necessarily provide better diversity

132 metrics (Devictor et al., 2010), and given that presence-absence data are more comparable

133 among sites than are abundance data (usually measured in different ways: number of nests,

134 individuals at baits, or individuals in pitfall traps), we focused our analyses on the presence-

135 absence dataset.

\section{Trait data}

137 We characterized 155 ant species according to 10 traits that determine different dimensions of

138 the ants' functional niches with respect to morphology, life-history, and behavior at both the

139 level of the individual worker and that of the colony (Tables S1 and S2). These traits are

140 considered important in ants because of their influence on ant autoecology and ecosystem

141 functioning (e.g., Hölldobler \& Wilson, 1990; Arnan et al., 2012, 2013, 2014); furthermore, they

142 strongly respond to environmental gradients (Arnan et al., 2012, 2013, 2014).

\section{Phylogenetic data}

144 We built a complete phylogeny for the 155 ant species considered (phylogenetic tree provided in

145 Fig. S1). This tree was the product of a super tree derived from a genus-level phylogeny created

146 using a molecular dataset (Moreau \& Bell, 2013). We then added species to this basal tree by

147 integrating the results of different studies, using a combination of molecular and taxonomic data

148 (Appendix S1). The tree was reconstructed with Mesquite version 3.0 (Maddison \& Maddison,

149 2014). For this phylogeny, reliable estimates of branch length and node ages were unavailable.

150 First, to solve the polytomies, we used 'multi2di' from the R (R Development Core Team, 2010) 
151 package 'phytools'. Second, the tree was ultrametrized applying Grafen's Rho transformation to

152 branch lengths, using the function 'compute.brlen' from the R package 'ape.'

\section{Environmental gradients}

154 Sites were classified according to their positions along different environmental gradients, which 155 were grouped into two broader gradients:

156 a) Climate gradients. Climate data for each site came from the WORLDCLIM database

157 (http://www.worldclim.org/bioclim); rasters of the highest available resolution (30 arc-seconds)

158 were used. We obtained values for four climate variables: mean annual temperature, temperature

159 amplitude (the difference between the maximum and the minimum annual temperatures), annual 160 precipitation, and precipitation seasonality (coefficient of variation of the monthly precipitation 161 level). Previous studies have highlighted the independence of these climate variables (Arnan et 162 al., 2014).

b) Land-use gradients. A land-use diversity index was calculated by applying the Simpson index of diversity to the percentage of different land-use categories within a 2-km radius area around the central point of each site. We used seven land-use categories (artificial surfaces, agricultural areas, forests, scrublands, meadows, wetlands, and water bodies), which were obtained from the CORINE land-cover vector database (Bossard et al., 2000), derived from 25-m resolution satellite data. Similarly, an anthropogenic disturbance index was calculated based on the proportion of the 2-km radius area around the center of each plot that was occupied by the

170 aforementioned artificial surfaces. We assumed that natural sites within areas with a higher

171 proportion of anthropogenic land-use would be more likely to suffer from anthropogenic 172 disturbance. 
173 All variables were standardized to have a mean of 0 and a variance of 1 (de Bello et al., 2010).

\section{Spatial structure}

175 In order to explore the sites' spatial structure, we generated a set of multiscale principal

176 coordinates of neighbor matrices (PCNM) from the geographic distance matrix using the $\mathrm{R}$

177 package $P C N M$. PCNM eigenfunctions depict a spectral decomposition of the spatial

178 relationships among sites. They are orthogonal sine waves that describe all the spatial scales that

179 can be accommodated in the sampling design (Dray et al. 2006), such that the first and last axes

180 represent broad- and fine-scale patterns, respectively. Sixty PCNMs were generated.

\section{Partitioning taxonomic, functional, and phylogenetic diversity}

182 To partition each facet of biodiversity considered (TD, FD, and PD) into alpha and beta components, we used the Rao quadratic entropy index, which provides a standardized methodology for comparing these components within the same mathematical framework

185 (Pavoine et al., 2004; de Bello et al., 2010; Devictor et al., 2010). Moreover, this index makes it possible to calculate functional diversity for combinations of traits, and it can handle quantitative, categorical, and binary traits (e.g., Rao, 1982; Leps et al., 2006). Furthermore, its estimates of functional and phylogenetic diversity are relatively independent of taxonomic diversity (e.g., Mouchet et al., 2010). We used additive partitioning to break down overall gamma diversity into within (alpha) and among (beta) community diversity. Within each community $k$ with $S$ species, $\alpha$-diversity was calculated using Rao's coefficient of diversity (Rao,

192 1982; Pavoine et al., 2004) modified for presence-absence data:

$$
\alpha \operatorname{Rao}_{(k)}=\sum_{i=1}^{S} \sum_{j=1}^{S} d_{i j}
$$


194 where $d_{i j}$ is the distance between species $i$ and $j$, which can be taxonomic, functional, or

195 phylogenetic. This index represents the expected dissimilarity between two individuals of

196 different species chosen randomly from the community. Between communities $k$ and $l, \beta$ -

197 diversity was computed using the Rao's dissimilarity index (Rao, 1982; Pavoine et al., 2004),

198 which is the expected distance between two individuals of different species chosen randomly

199 from two distinct communities:

200

$$
\beta \text { Rao }_{\text {pairwise }(k, l)}=\left(\gamma_{(k+l)}-\bar{\alpha}_{(k, l)}\right) / \gamma_{(k+l)}
$$

201

202

where $\gamma_{\mathrm{k}+1}$ is the gamma diversity of the pair of communities (calculated with the same equation as for alpha diversity, but taking into account all the species included in the two communities) and $\bar{\alpha}_{(\mathrm{k}, \mathrm{l})}$ is the mean $\alpha$-diversity of the two communities. Prior to performing the calculations, we applied Jost's correction (Jost, 2007) to $\gamma$ and $\alpha$ to properly quantify $\beta$-diversity independently of $\alpha$-diversity (de Bello et al., 2010). To carry out these calculations, we used the function 'rao' (de Bello et al., 2010) in R.

To calculate the Rao quadratic entropy index, different distance measures were used depending on the facet of diversity considered. Taxonomic distances between species were measured as $\mathrm{d}_{i j}=1$ when $i \neq j$, and $\mathrm{d}_{i j}=0$ when $i=j$. To compute functional distances between species, we first conducted a principal component analysis $(\mathrm{PCA})$ on the standardized (mean=0, $\mathrm{SD}=1$ ) trait data to correct for dominance in the distance matrix by highly correlated traits (Devictor et al., 2010; Purschke et al., 2013). The resulting PCA axes were used to calculate Euclidean distances. Phylogenetic distances between species were measured with the cophenetic distances from the phylogenetic tree. We scaled all distances between 0 and 1 by dividing each type of distance by 
215 its maximum value in order to make taxonomic, functional, and phylogenetic distances

216 comparable.

\section{Statistical analyses}

218 Moran's I and Mantel tests were used to test for spatial autocorrelation between the alpha and

219 beta components of TD, $\mathrm{PD}$, and $\mathrm{FD}$, respectively.

220 We used redundancy analysis (RDA) with variation partitioning (Borcard et al., 1992) to assess

221 the relative influence of environmental and spatial factors, alone and combined, on alpha- and

222 beta-level variation in TD, PD, and FD. We partitioned the variation into multiple components: a

223 pure environmental component, a pure spatial component, a spatially structured environmental

224 component, and residual variation. Forward selection (Blanchet et al., 2008) was used for each

225 set of environmental and spatial variables to select only those variables that significantly

226 explained variation in the dependent variables ( $\mathrm{p}<0.05$, after 999 random permutations). Only the

227 selected variables were used in variation partitioning. $R^{2}$ values adjusted for the number of sites

228 and explanatory variables were used throughout because they provided corrected estimates of

229 explained variation ( $\mathrm{R}^{2}$ adj; Peres-Neto et al., 2006). Monte Carlo permutation tests (9999

230 permutations) were used to calculate the significance levels of the different components. Since

231 the beta diversity indices estimated with the RaoQ index were dissimilarity matrices, they cannot

232 be used directly as response variables in this type of variation-partitioning framework. Therefore,

233 prior to conducting the RDA analysis and variation partitioning, we transformed the dissimilarity

234 matrices into data frames by conducting a principal coordinate analysis $(\mathrm{PCoA})$ on the

235 dissimilarity matrices; we then used the scores of the significant axes as the representative values

236 for each community (Legendre \& Anderson, 1999). Mixing traits in the functional diversity

237 index as we did could have resulted in FD demonstrating a neutral response to gradients; this 
238 does not occur when individual traits are used. To address this problem, we also conducted

239 analyses of FD for each trait separately.

240 A relationship between FD and PD could be explained by significant phylogenetic signals in the

241 traits used to calculate FD. We therefore tested for their presence using Pagel's $\lambda$ test (Pagel,

242 1999), which assumes a Brownian motion (BM) model of trait evolution. To test for statistical

243 significance, we used a likelihood ratio test approximated by a chi-squared distribution to

244 compare the negative log likelihood obtained when there is no signal (i.e., using the tree

245 transformed $\lambda=0$ ) to that estimated from the original topology.

246 All analyses were conducted in R using the packfor, PCNM, vegan, ade4, and Geiger packages.

\section{RESULTS}

We found strong autocorrelations between both the alpha and beta components in the three facets of diversity (Figure 2). According to our first hypothesis, our RDA analyses revealed that both environmental and spatial factors significantly contributed to variation in alpha- and beta-level TD, PD, and FD (Figure 3). Furthermore, we found that spatial factors made a much larger contribution than environmental factors for all dependent variables (Figure 3), which indicates that ant communities within our study area were strongly structured by space. The PCNMs retained in the alpha- and beta-level models of spatial structure were mostly broad in scale (Figure 4).

Although environmental factors made a relatively smaller contribution, they nonetheless appear to play an important role in structuring ant diversity. Interestingly, the different facets of diversity mostly responded to similar environmental gradients in the same way (Table 1), which 
260 gives support to our second hypothesis. In the alpha-level RDAs, two of the six environmental

261 variables (mean annual temperature and anthropogenic disturbance) were retained in the TD, PD,

262 and FD models, which highlight the role of these two factors in determining ant diversity. In

263 particular, warmer and less disturbed sites had higher levels of all three types of diversity. Mean

264 annual temperature explained most of the variation in PD and FD; in the case of TD,

265 anthropogenic disturbance explained an equivalent amount of variation. TD and FD were

266 positively correlated with temperature amplitude, and TD and FD were negatively correlated

267 with precipitation seasonality and annual precipitation, respectively (Table 1). No facet of

268 diversity was influenced by land-use diversity. In the beta-level RDAs, four of the six

269 environmental variables (mean annual temperature, temperature amplitude, precipitation

270 seasonality, and anthropogenic disturbance) were retained in the TD, PD, and FD models (Table

271 1). More specifically, the greater the distance among these environmental variables, the higher

272 the turnover in TD, PD, and FD. Once again, mean annual temperature explained most of the

273 variation. Turnover in TD was mediated by differences in annual precipitation, while turnover in

274 FD was mediated by annual precipitation and land-use diversity (Table 1).

275 At the alpha scale, environmental factors accounted for only a small fraction of the total variation 276 in TD (3\%), PD (1\%), and FD (6\%). Spatial factors had greater explanatory ability $(21,27$, and

$27721 \%$, respectively). Spatially structured environmental factors explained 13, 16, and $23 \%$ of the

278 total variation in TD, PD, and FD, respectively (Figure 3). Environmental factors (alone, and in

279 tandem with space) made a greater contribution to FD than to TD and PD. These results are in

280 agreement with our third hypothesis. Like at the alpha scale, at the beta scale, environmental

281 factors accounted for only a small fraction of the total variation in TD (6\%), PD (4\%), and FD

282 (5\%). Spatial factors explained 21,18 , and $15 \%$ of the variation in TD, PD, and FD, respectively. 
283 In contrast, spatially structured environmental factors had the greatest explanatory ability (TD:

$28441 \%$, PD: 24\%, and FD: 39\%, respectively). Environmental factors (alone, and in tandem with

285 space) made a greater contribution to TD and FD than to PD. Overall, they explained much more

286 beta- than alpha-level variation in TD, PD, and FD, especially in the case of TD and FD (Figure 287 3).

288 When the functional traits were examined separately (Table S3, Fig. S2), it was clear that FD

289 was shaped by individual trait responses at both the alpha and beta scales. Traits responded

290 differently (positively or negatively) to the various environmental gradients and made different

291 contributions to the environmental and spatial components. Indeed, the global FD pattern did not

292 seem to be driven by any particular trait, since no single trait showed the same pattern as global

293 FD. Furthermore, individual functional responses did not mirror TD and FD patterns at either the

294 alpha or the beta scale. However, all the functional traits had significant phylogenetic signals

295 (Table S4), which is evidence (albeit not definitive) for niche conservatism. The values of

296 Pagel's $\lambda$ ranged from 0.78 for polydomy to 1 for diet (seeds, in particular) and foraging strategy,

297 which demonstrates that phylogenetic signals were strong for most of the functional traits.

\section{DISCUSSION}

300

In accordance with our first hypothesis, we found that environmental factors and spatial factors separately influence alpha- and beta-level TD, PD, and FD. In particular, it seems that climate and human-modified landscapes shape the different facets of ant diversity in western and central Europe. More interestingly, spatial factors played a large role, which suggests that dispersal limitations have a strong effect on ant community structure at the different diversity levels. 
may reflect the omission of certain spatially structured environmental variables (Jones et al., 2008). We refute this interpretation for several reasons. First, we examined a wide range of key gradients, including the main climate and human-modified landscape gradients in western and central Europe. Other studies have found that similar gradients have an influence on the structure of animal communities (Meynard et al., 2011), including ant communities (Arnan et al., 2012, 2014). Second, there were strong spatial autocorrelations in all the diversity metrics (Figure 1), and we found that alpha- and beta-level spatial structure in all the diversity facets was best explained by broad-scale PCNMs (Figure 4). Third, our study area encompassed much of western and central Europe, which contains a great diversity of habitats, and even spanned across some large mountain ranges. Fourth, ant gynes seem to face dispersal limitations as a general rule (Mezger \& Pfeiffer, 2011) because ants are small and their alates are often poor dispersers. Altogether, this evidence suggests that, given the presence of mountain ranges, habitat diversity, and other obstacles, dispersal limitations strongly structure ant communities across Europe at all diversity levels. In this respect, our results agree with those of Meynard et al. (2011), who found that spatial structure played a preponderant role in structuring alpha- and beta-level TD, PD, and FD in French bird communities. Dispersal limitations are much more severe in ants than in birds, and, accordingly, our study found a stronger effect of spatial factors on diversity.

Although we found that ant diversity patterns in western and central Europe are determined by spatial and environmental parameters, our results also highlight a large portion of unexplained variation in alpha and beta diversity patterns. This fact suggests that other processes are at work in determining ant diversity patterns. For instance, ant diversity patterns across Europe might also be determined by stochastic mechanisms, assuming that population dynamics do not depend on environmental characteristics and are primarily driven by ecological drift and dispersal 
(Hubbell, 2001). Also, the omission of non-spatially structured biological or environmental variation might also account for part of the unexplained variance. For instance, it is known the role of competitive interactions in structuring ant diversity at the local scale (Cerdá et al. 2013); the fact that the portion of unexplained variance is higher for the alpha than for the beta diversity (Figure 3), might suggest that variation in competitive interactions along the gradients might account for part of the unexplained variance in ant diversity patterns.

We also found largely support for our second hypothesis: TD, PD, and FD in ant communities demonstrated parallel responses along most of the environmental gradients in western and central Europe. Given that spatially structured environmental factors had strong effects (Figure 1), a possible explanation is that topography-related dispersal limitations affect particular functional groups and/or lineages, and consequently, particular species. It is worth mentioning here that we found strong phylogenetic signals in the traits we examined (Table S3), which might explain why PD and FD showed highly similar responses. Once again, our results corroborated those of Meynard et al. (2011), the only other study conducted thus far that had similar aims and a comparably large spatial scale. The authors found general support for the idea that hypotheses generated for local and regional TD can be extended to PD and FD. Conversely, Bernard-Verdier et al. (2013) observed no congruence among alpha- and beta-level TD, PD, and FD along local gradients of soil type and resource availability. Purschke et al. (2013) found similar incongruence in a plant community over the course of plant succession. A recent study that analyzed the FD of plants along a latitudinal gradient in the New World found that patterns of alpha-, beta-, and gamma-level diversity failed to match any one theory of species diversity 
351 are somewhat related and concluded that any mismatches were attributable to environmental

352 factors (Safi et al., 2011).

353 However, we also found some environment-mediated mismatches, which might be due to

354 assorted environmental drivers differentially filtering the different facets of diversity (Safi et al.,

355 2011; Hermant et al., 2012). For instance, diversity patterns varied along the annual precipitation

356 gradient. Precipitation usually influences primary productivity and resource availability (Leith \&

357 Whittaker, 1975). The lower functional diversity in wetter areas might stem from relaxed local

358 competition: higher levels of primary productivity could mean less competition for resources and

359 thus lower rates of species extinction, ultimately resulting in functional redundancy (Pavoine \&

360 Bonsall, 2011). Consequently, functional turnover should be much higher than taxonomic

361 turnover along the precipitation gradient, which is what we observed. Moreover, the degree of

362 niche conservatism might vary for different environmental gradients, which might explain why

363 patterns among the three facets of diversity differed in some gradients. However, this speculation

364 remains outside the scope of our paper.

365 In our study, the most striking environmental gradient was a climate gradient, along which mean

366 annual temperature varied: it influenced all three facets of diversity and explained much more

367 variation than the other environmental factors. This finding highlights the role of temperature as

368 one of the main drivers of biodiversity, which supports concerns about the effects of climate

369 change on species distributions (Dunn et al., 2009; Jenkins et al., 2011), as well as related

370 ecosystem services and evolutionary responses. The negative effect that anthropogenic

371 disturbance had on all three facets of diversity at both alpha and beta scales - triggering diversity

372 turnover - should also be underscored. Furthermore, land-use diversity affected functional

373 turnover. These findings are noteworthy given concerns about the functional consequences of 
374 current biodiversity losses (Loreau et al., 2001), especially those mediated by human-driven

375 changes (Foley et al., 2005); indeed, the most important driver of declining biodiversity is

376 changes in land use (Sala et al., 2000).

377 In accordance with our third hypothesis, FD responded to more environmental gradients than

378 either TD or PD did. Furthermore, when all three facets of diversity significantly responded to

379 the same environmental gradient, relatively more variation in FD was explained. However, our

380 single-trait analyses suggest that this finding might be contingent on the trait examined. At any

381 rate, the large contribution of environmental gradients to the multi-trait FD index seems

382 somewhat obvious, because a species' traits clearly determine whether it will successfully pass

383 through an environmental filter (Pavoine et al., 2011) and consequently are the underlying force

384 shaping functional composition. Interestingly, environmental factors explained a similar amount

385 of beta-level variation in TD and FD, which suggests that strong environmental filtering is

386 operating along these gradients (Mouchet et al., 2010). If species sorting is weak, we would not

387 expect to see major changes in functional traits, i.e., we would not expect high functional

388 turnover even if species turnover occurred. In contrast, if there is strong species sorting along

389 environmental gradients, we would expect both species and functional turnover (Mouchet et al.,

390 2010).

391 Notably, environmental factors explained more alpha- and beta-level variation in FD than in PD.

392 In birds, Meynard et al. (2011) found the opposite pattern; they attributed this finding to either

393 overlooking some of the relevant gradients that affect FD, using poorly chosen life-history traits

394 to measure FD, or the fact that PD is simply a more integrative proxy than FD when analyzing a

395 given subset of life-history traits. In this study, we refute the general assumption that PD is a

396 more integrative measure of FD (Cadotte et al., 2009; Meynard et al., 2011), and we give 
empirical support to those authors who have questioned this assumption from a theoretical standpoint (Losos, 2008; Cavender-Bares et al., 2009; Mouquet et al., 2012). Phylogenetic diversity does not appear to be a good proxy for FD when a large number of well-chosen, diverse functional traits are used. We selected morphological, life-history, and behavioral traits related to resource exploitation, reproduction, and social structure; these traits have been demonstrated to be strongly related to the abiotic environment (Arnan et al., 2013, 2014) and may therefore reflect effective local adaptation. Our results lend credence to the idea that FD is a better indicator of community assembly processes than PD (Díaz et al., 2007).

We also found that environmental factors had a stronger effect on TD, PD, and FD at the beta level than at the alpha level, which supports the idea that environmental filtering is stronger at the regional scale (Cornwell et al., 2006). Other kinds of mechanisms, such as species interactions (e.g., competition or facilitation), might have larger effects at the local scale than at the regional scale (Cavender-Bares et al., 2004, Slingsby \& Verboom, 2006). Moreover, beta diversity responded more similarly to environmental gradients than did alpha diversity. This finding concurs with the results of some past work (Devictor et al., 2010; Bernard-Verdier et al., 2013), which found relatively greater congruence among different facets of beta-level vs. alphalevel diversity.

Finally, we should assume that our study might have some limitations, mainly related to the fact that the communities we used are not evenly distributed across the spatial coverage of this study, and they account for a subset of species known to occur in western and central Europe. However, our study encompasses the most comprehensive dataset on ant communities in Europe. Although our communities do not display a regular spatial distribution, they encompassed most of the range that the environmental variables take across western and central Europe; moreover, the 
420 species we found in this study are the most common species of the region. All this suggest that

421 the results from our sampled communities are representative of the patterns of ant diversity in

422 western and central Europe.

\section{Conclusions}

424 By using variation-partitioning analyses, we have demonstrated that ant diversity patterns in western and central Europe, whether TD, PD, or FD, are driven by both environmental determinism and dispersal limitations, with the latter playing a more prominent role. The strong autocorrelations that we found in our diversity data, along with the potent effects of dispersal limitations, underscore that TD, PD, and FD are highly heterogeneous in western and central Europe. This finding implies that western and central European ant communities are taxonomically, phylogenetically, and functionally clustered (Zupan et al., 2014), which might be the result of historical and/or evolutionary forces. For instance, the study area is composed of highly diverse biogeographic regions, which might display different rates of trait evolution and speciation (e.g., Weir \& Schluter, 2007; Cooper \& Purvis, 2010). Recent massive diversification events (Slingsby \& Verboom, 2006), and the different historical disturbance regimes at the origin of current-day European landscapes (Schelaas et al., 2003) might contribute to this heterogeneity in ant diversity. Our findings also highlight that the different facets of diversity are fairly equivalent because they demonstrate similar patterns along environmental gradients. However, environmental factors explained the most variation in FD, which reflects the strong effect that species sorting (i.e., environmental filtering) has on these patterns. Furthermore, functional traits

440 had strong phylogenetic signals, which suggests that niche conservatism might account for the 441 parallel patterns displayed by FD and PD and, therefore, also have relevance for diversity 442 patterns in general. It is clear that incorporating phylogenetic relationships and functional 
443 ecology into analyses of ecological patterns allowed us to draw stronger conclusions regarding

444 the mechanisms that underlie macroecological patterns at different spatial scales (Webb et al.,

445 2002; Cavender-Bares et al., 2009). There is thus a definite need to integrate the information

446 furnished by different facets of diversity to better understand the assembly rules responsible for 447 current global patterns of biodiversity.

\section{ACKNOWLEDGMENTS}

450 We are very grateful to Alejandro González-Voyer and Iván Gómez-Mestre for their help with 451 the phylogeny, Bernhard Seifert and Laszlo Gallé for providing their data on central European 452 ant communities and Jessica Pearce-Duvet for her English editing services.

\section{REFERENCES}

455

456

457

458

459

460

461

462 463

Arnan X, Cerdá X, Retana J. 2012. Distinctive life traits and distribution along environmental gradients of dominant and subordinate Mediterranean ant species. Oecologia 170:489-500.

Arnan X, Cerdá X, Rodrigo A, Retana J. 2013. Response of ant functional composition to fire. Ecography 36:1182-1192.

Arnan X, Cerdá X, Retana J. 2014. Ant functional responses along environmental gradients. Journal of Animal Ecology 83:1398-1408.

Bernard-Verdier M, Flores O, Navas M, Garnier E. 2013. Partitioning phylogenetic and functional diversity into alpha and beta components along an environmental gradient in a Mediterranean rangeland. Journal of Vegetation Science 24:877-889. 
464 Blanchet FG, Legendre P, Borcard D. 2008. Forward selection of explanatory variables. Ecology $465 \quad 89: 2623-2632$.

466 Borcard D, Legendre P, Drapeau P. 1992. Partialling out the spatial component of ecological 467 variation. Ecology 73:045-1055.

468 Borcard D, Legendre P, Avois-Jacquet P, Tuomisto H. 2004. Dissecting the spatial structure of 469 ecological data at multiple scales. Ecology 85:1826-1832.

470 Bossard M, Feranec J, Otahel J. 2000. CORINE land cover technical guide - addendum 2000.

471 Technical Report no. 40. European Environment Agency, Copenhagen.

472 Cadotte MW, Cavender-Bares J, Tilman D, Oakley TH. 2009. Using phylogenetic, functional 473 and trait diversity to understand patterns of plant community productivity. PLoS ONE 4:e5695.

474 Cavender-Bares J, Kozak KH, Fine PVA, Kembel SW. 2009. The merging of community 475 ecology and phylogenetic biology. Ecology Letters 12:693-715.

476 Cerdá X, Arnan X, Retana J. 2013. Is competition a significant hallmark of ant (Hymenoptera:

477 Formicidae) ecology? Myrmecological News 18:131-147.

478 Cooper N, Purvis A. 2010. Body size evolution in mammals: complexity in tempo and mode. 479 American Naturalist 175:727-738.

480 Cornwell WK, Schwilk DW, Ackerly DD. 2006. A trait-based test for habitat filtering: convex 481 hull volume. Ecology 87:1465-1471.

482 De Bello F, Lavergne S, Meynard CN, Leps J, Thuiller W. 2010. The partitioning of diversity: 483 showing Theseus a way out of the labyrinth. Journal of Vegetation Science 21:992-1000. 
484

485

486

487

488

489

490

491

492

493

494

495

496

497

498

499

500

501

502

503

504

505

506

Devictor V, Mouillot D, Meynard C, Jiguet F, Thuiller W, Mouquet N. 2010. Spatial mismatch and congruence between taxonomic, phylogenetic and functional diversity: the need for integrative conservation strategies in a changing world. Ecology Letters 13:1030-1040.

Díaz S, Lavorel S, de Bello F, Quétier F, Grigulis K, Robson M. 2007. Incorporating plant functional diversity effects in ecosystem service assessments. Proceedings of the National Academy of Sciences of the United States of America 104:20684- 20689.

Dray S, Legendre P, Peres-Neto PR. 2006. Spatial modeling: a comprehensive framework for principal coordinate analysis of neighbor matrices (PCNM). Ecological Modelling 196:483-493. Dunn RR, Agosti D, Andersen AN, Arnan X, Bruhl CA, Cerdá X, Ellison AM, Fisher BL, Fitzpatrick MC, Gibb H, Gotelli NJ, Gove AD, Guenard B, Janda M, Kaspari ME, Laurent EJ, Lessard JP, Longino JT, Majer JD, Menke SB, McGlynn TP, Parr CL, Philpott SM, Pfeiffer M, Retana J, Suarez AV, Vasconcelos HL, Weiser MD, Sanders NJ.. 2009. Climatic drivers of hemispheric asymmetry in global patterns of ant species richness. Ecology Letters 12:324-333. Faith DP. 2008. Threatened species and the potential loss of phylogenetic diversity: conservation scenarios based on estimated extinction probabilities and phylogenetic risk analysis.

Conservation Biology 22:1461-1470.

Foley JA, DeFries R, Asner GP, Barford C, Bonan G, Carpenter SR, Chapin FS, Coe MT, Daily GC, Gibbs HK, Helkowski JH, Holloway T, Howard EA, Kucharik CJ, Monfreda C, Patz JA, Prentice IC, Ramankutty N, Snyder PK. 2005. Global consequences of land-use. Science 309:570-574.

Forest F, Grenyer R, Rouget M, Davies TJ, Cowling RM, Faith DP, Balmford A, Manning JC, Procheş S, van der Bank M, Reeves G, Hedderson TA, Savolainen V. 2007. Preserving the evolutionary potential of floras in biodiversity hotspots. Nature 445:757-760. 
507 Fox JW. 2013. The intermediate disturbance hypothesis should be abandoned. Trends in Ecology

508 and Evolution 28:86-92.

509 Gaston KJ. 1996. Biodiversity - latitudinal gradients. Progress in Physical Geography 20:466510476.

511 Hermant M, Hennion F, Bartish I, Yguel B. 2012. Disparate relatives: life histories vary more in 512 genera occupying intermediate environments. Perspectives in Plant Ecology, Evolution and 513 Systematics 14:283-301.

514 Hölldobler B, Wilson EO. 1990. The ants. Harvard University Press, Cambridge.

515 Hubbell SP. 2001. The Unified Neutral Theory of Biodiversity and Biogeography. Princeton 516 University Press, Princeton.Jenkins CN, Sanders NJ, Andersen AN, Arnan X, Brühl A, Cerdá X, 517 Ellison AM, Fisher BL, Fitzpatrick MC, Gotelli NJ, Gove AD, Guénard B, Lattke JE, Lessard 518 JP, McGlynn TP, Menke SB, Parr CL, Philpott SM, Vasconcelos HL, Weiser MD, Dunn RR. 519 2011. Global diversity in light of climate change: the case of ants. Diversity and Distributions $520 \quad 17: 652-662$.

521 Jones MM, Tuomisto H, Brocard D, Legendre P, Clark DB, Olivas PC. 2008. Explaining

522 variation in tropical plant community composition: influence of environment and spatial data 523 quality. Oecologia 155:593-604.

524 Jost L. 2007. Partitioning diversity into independent alpha and beta components. Ecology 88: $525 \quad 2427-2439$.

526 Kraft NJB, Cornwell WK, Webb CO, Ackerly DD. 2007. Trait evolution, community assembly, 527 and the phylogenetic structure of ecological communities. American Naturalist 170:271-283. 
528 Lamanna C, Blonder B, Violle C, Kraft NJ, Sandel B, Š́mová I, Donoghue JC, Svenning

529 JC, McGill BJ, Boyle B, Buzzard V, Dolins S, Jørgensen PM, Marcuse-Kubitza A, Morueta-

530 Holme N, Peet RK, Piel WH, Regetz J, Schildhauer M, Spencer N, Thiers B, Wiser SK, Enquist

531 BJ. 2014. Functional trait space and the latitudinal gradient. Proceedings of the National

532 Academy of Sciences of the USA 111:13745-13750.Legendre P, Anderson MJ. 1999. Distance-

533 based redundancy analysis: testing multispecies responses in multifactorial ecological

534 experiments. Ecological Monographs 69:1-24.

535 Legendre P, Legendre L. 1998. Numerical Ecology, 2nd Edition. Elsevier Science, Amsterdam.

536 Legendre P, Mi X, Ren H, Ma K, Yu M, Sun I-F, He F. (2009) Partitioning beta diversity in a

537 subtropical broad-leaved forest of China. Ecology 90:663-674.

538 Leith H, Whittaker RH. 1975. Primary Productivity of the Biosphere. New York: Springer-

539 Verlag.

540 Lepš J, de Bello F, Lavorel S, Berman S. 2006. Quantifying and interpreting functional diversity

541 of natural communities: practical considerations matter. Preslia 78:481-501.

542 Loreau M, Naeem S, Inchausti P, Bengtsson J, Grime JP, Hector A, Hooper DU, Huston

543 MA, Raffaelli D, Schmid B, Tilman D, Wardle DA. 2001. Biodiversity and ecosystem

544 functioning: current knowledge and future challenges. Science 294:804-808.

545 Losos JB. 2008. Phylogenetic niche conservatism phylogenetic signal and the relationship

546 between phylogenetic relatedness and ecological similarity among species. Ecology Letters

547 11:995-1003.

548 Maddison WP, Maddison DR. 2014. Mesquite: a modular system for evolutionary analysis.

549 Version 3.0. http:/mesquiteproject.org 
550

551

552

553

554

555

556

557

558

559

560

561

562

563

564

565

566

567

568

569

Meynard CN, Devictor V, Mouillot D, Thuiller W, Jiguet F, Mouquet N. 2011. Beyond

taxonomic diversity patterns. How do $\alpha, \beta$ and $\gamma$ components of bird functional and phylogenetic diversity respond to environmental gradients across France? Global Ecology and Biogeography 20:893-903.

Mezger D, Pfeiffer M. 2011. Partitioning the impact of abiotic factors and spatial patterns on species richness and community structure of ground ant assemblages in four Bornean rainforests. Ecography 34:39-48.

Moreau CS, Bell CD. 2013. Testing the museum versus cradle biological diversity hypothesis:

Phylogeny, diversification, and ancestral biogeographic range evolution of the ants. Evolution 67:2240-2257.

Mouchet MA, Villéger S, Mason NWH, Mouillot D. 2010. Functional diversity measures: an overview of their redundancy and their ability to discriminate community assembly rules.

Functional Ecology 24:867-876.

Mouquet N, Devictor V, Meynard CN, Munoz F, Bersier LF, Chave J, Couteron P, Dalecky A, Fontaine C, Gravel D, Hardy OJ, Jabot F, Lavergne S, Leibold M, Mouillot D, Münkemüller T, Pavoine S, Prinzing A, Rodrigues AS, Rohr RP, Thébault E, Thuiller W. 2012. Ecophylogenetics: advances and perspectives. Biological Reviews 87:769-785.

Pagel M. 1999. Inferring the historical patterns of biological evolution. Nature, 401, 877-884. Pavoine S, Bonsall MB. 2011. Measuring biodiversity to explain community assembly: a unified approach. Biological Reviews 86:792-812. 
570 Pavoine S, Dufuor AB, Chessel D. 2004. From dissimilarities among species to dissimilarities

571 among communities: a double principal coordinate analysis. Journal of Theoretical Biology,

$572 \quad$ 228:523-537.

573 Pavoine S, Vela E, Gachet S, de Bélair G, Bonsall, M.B. (2011) Linking patterns in phylogeny,

574 traits, abiotic variables and space: a novel approach to linking environmental filtering and plant

575 community assembly. Journal of Ecology 99:165-175.

576 Peres-Neto PR, Legendre P, Dray S, Borcard D. 2006. Variation partitioning of species data

577 matrices: estimation and comparison of fractions. Ecology 87:2614-2625.

578 Petchey OL, Gaston KJ. 2006. Functional diversity: back to basics and looking forward. Ecology

579 Letters 9:741-758.

580 Pianka ER. 1966. Latitudinal gradients in species diversity: a review of concepts. American

581 Naturalist 100:33-46.

582 Purschke O, Schmid BC, Sykes MT, Poschlod P, Michalski SG, Durka W, Kühn I, Winter M,

583 Prentice HC. 2013. Contrasting changes in taxonomic, phylogenetic and functional diversity

584 during a long-term succession: insights into assembly processes. Journal of Ecology 101:857585866.

586 R Development Core Team. 2010. $R$ : a language and environment for statistical computing. $\mathrm{R}$

587 Foundation for Statistical Computing, Vienna, Austria. http://www.R-project.org.

588 Rahbek C, Gotelli NJ, Colwell RK, Entsminger GL, Rangel FLVB, Graves GR. 2007. Predicting

589 continental-scale patterns of bird species richness with spatially explicit models. Proceedings of

590 the Royal Society B 274:165-174. 
591 Rao CR. 1982. Diversity and dissimilarity coefficients: a unified approach. Theoretical

592 Population Biology 21:24-43.

593 Safi K, Cianciaruso MV, Loyola RD, Brito D, Armour-Marshall K, Diniz-Filho JAF. 2011.

594 Understanding global patterns of mammalian functional and phylogenetic diversity.

595 Philosophical Transactions of the Royal Society B 366:2536-2544.

596 Sala OE, Chapin FS, Armesto JJ, Berlow E, Bloomfield J, Dirzo R, Huber-Sanwald E, Huenneke

597 LF, Jackson RB, Kinzig A, Leemans R, Lodge DM,Mooney HA, Oesterheld M, Poff NL, Sykes

598 MT, Walker BH, Walker M, Wall DH. 2000. Global biodiversity scenarios for the year 2100.

599 Science 287:1770-1774.

600 Schelhaas M-J, Nabuurs G-J, Schuck A. 2003. Natural disturbances in the European forests in 601 the 19th and 20th centuries. Global Change Biology 9:1620- 1633.

602 Slingsby JA, Verboom GA. 2006. Phylogenetic relatedness limits co-occurrence at fine spatial 603 scales: evidence from the Schoenoid sedges (Cyperaceae: Schoeneae) of the Cape Floristic 604 Region, South Africa. American Naturalist 168:14-27.

605 Tuomisto H, Ruokolainen K, Yli-Halla M. 2003. Dispersal, environment, and floristic variation 606 of western Amazonian forests. Science 299:241-244.

607 Webb CO, Ackerly DB, McPeek MA, Donoghue MJ. 2002. Phylogenies and community 608 ecology. Annual Review of Ecology, Evolution and Systematics 33:475-505.

609 Weir JT, Schluter D. 2007. The latitudinal gradient in recent speciation and extinction rates of 610 birds and mammals. Science 315:1574-1576.

611 Whittaker RJ, Willis KJ, Field R. 2001. Scale and species richness: towards a general

612 hierarchical theory of species diversity. Journal of Biogeography 28:453-470. 
613 Willig MR, Kaufman DM, Stevens RD. 2003. Latitudinal gradients of biodiversity: pattern, 614 process, scale, and synthesis. Annual Review of Ecology, Evolution and Systematics 34:273-309.

615 Zupan L, Cabeza M, Maiorano L, Roquet C, Devictor V, Lavergne S, Mouillot D, Mouquet N, 616 Renaud J, Thuiller W.2014. Spatial mismatch of phylogenetic diversity across three vertebrate 617 groups and protected areas in Europe. Diversity and Distributions 20:674-685. 
619 Figure 1. Map of the study area showing plot distribution.

620 Figure 2. Correlograms for the alpha and beta components of the taxonomic, phylogenetic and 621 functional diversity. We used the Moran's I and Mantel tests for alpha and beta diversity, 622 respectively. Black circles indicate significant correlation $(\mathrm{p}<0.05)$.

623 Figure 3. Partitioning of variation in alpha- and beta-level taxonomic (TD), phylogenetic (PD), 624 and functional (FD) diversity. The figure depicts the adjusted unique contribution $\left(\mathrm{R}^{2}\right)$ of 625 environmental factors (light gray), spatial factors (black), spatially structured environmental 626 factors (dark gray), and unexplained variation (white), as calculated in the RDA analyses. Tests 627 of significance for the environmental and spatial factors were all significant $(\mathrm{p}<0.05)$.

628 Figure 4. Selected PCNMs from the 60 PCNMs that exhibit a positive spatial correlation for the 629 alpha and beta components of taxonomic (TD), phylogenetic (PD) and functional (FD) diversity. 630 The selected PCNMs from forward selection analyses are represented by red circles. 